\title{
SOME STABILITY THEOREMS FOR NONHARMONIC FOURIER SERIES
}

\author{
ROBERT M. YOUNG ${ }^{1}$
}

\begin{abstract}
The theory of nonharmonic Fourier series in $L^{2}(-\pi, \pi)$ is concerned with the completeness and expansion properties of sets of complex exponentials $\left\{e^{i \lambda_{n} t}\right\}$. It is well known, for example, that the completeness of the set $\left\{e^{i \lambda_{n} t}\right\}$ ensures that of $\left\{e^{i \mu_{n} t}\right\}$ whenever $\sum\left|\lambda_{n}-\mu_{n}\right|<\infty$. In this note we establish two results which guarantees that if $\left\{e^{i \lambda_{n} t}\right\}$ is a Schauder basis for $L^{2}(-\pi, \pi)$, then $\left\{e^{i \mu_{n} t}\right\}$ is also a Schauder basis whenever $\left\{\mu_{n}\right\}$ is "sufficiently close" to $\left\{\lambda_{n}\right\}$.
\end{abstract}

1. Introduction. The theory of nonharmonic Fourier series in $L^{2}(-\pi, \pi)$ is concerned with the completeness and expansion properties of sets of complex exponentials $\left\{e^{i \lambda_{n} t}\right\}$. It is well known, for example, that the completeness of the set $\left\{e^{i \lambda_{n} t}\right\}$ ensures that of $\left\{e^{i \mu_{n} t}\right\}$ whenever $\sum\left|\lambda_{n}-\mu_{n}\right|<\infty$ [1] (see also [5]). In this note a stronger result is established under the added hypothesis that $\left\{e^{i \lambda_{n} t}\right\}$ is a Schauder basis (compare [14, Theorem 2]).

THEOREM 1. Let $\left\{\lambda_{n}\right\}$ be a sequence of points lying in a strip parallel to the real axis. If $\left\{e^{i \lambda_{n} t}\right\}$ is a Schauder basis for $L^{2}(-\pi, \pi)$, then $\left\{e^{i \mu_{n} t}\right\}$ is also a Schauder basis whenever $\sum\left|\lambda_{n}-\mu_{n}\right|^{2}<\infty$.

One of the fundamental results from the theory of nonharmonic series states that the functions $e^{i \lambda_{n} t}(-\infty<n<\infty)$ form a Besselian basis for $L^{2}(-\pi, \pi)$ if they satisfy an inequality of the form

$$
\left\|\sum c_{n}\left(e^{i \lambda_{n} t}-e^{i n t}\right)\right\|^{2} \leqslant \theta^{2} \sum\left|c_{n}\right|^{2}
$$

for some $\theta(0 \leqslant \theta<1)$ and all finite sequences $\left\{c_{n}\right\}[9$, p. 109]. By "Besselian" is meant that each function $f$ in the space has a unique representation $f=\sum c_{n} e^{i \lambda_{n} t}$ with $\sum\left|c_{n}\right|^{2}<\infty$. It is well known [3] (see also [4]) that condition (1) holds whenever

$$
\left|\lambda_{n}-n\right| \leqslant L<(\log 2) / \pi \quad(-\infty<n<\infty) .
$$

Received by the editors May 15, 1975 and, in revised form, November 3, 1975.

AMS (MOS) subject classifications (1970). Primary 46B15; Secondary 46E30.

Key words and phrases. Nonharmonic Fourier series, Besselian basis, entire functions of exponential type.

1 Acknowledgement. The author wishes to thank Eric Margolis for his many helpful suggestions. 
In another direction, Levinson showed [8, p. 48] that if

$$
\left|\lambda_{n}-n\right| \leqslant L<1 / 4
$$

then every function in $L^{2}(-\pi, \pi)$ has a nonharmonic series expansion $f$ $\sim \sum c_{n} e^{i \lambda_{n} t}$ which is equiconvergent with its ordinary Fourier series over any interval $[-\pi+\varepsilon, \pi-\varepsilon]$ for any positive $\varepsilon$. For $\lambda_{n}$ real, the question of whether (2) implies (1) was answered in the affirmative by M. I. Kadec [7].

Using an interpolation property for entire functions of exponential type, we are able to show, more generally, that any Besselian basis of complex exponentials can be suitably perturbed. Specifically, we have the following result (compare [13, Theorem A, p. 412]).

THEOREM 2. Let $\left\{\lambda_{n}\right\}$ be a sequence of real numbers such that $\left\{e^{i \lambda_{n} t}\right\}$ is a Besselian basis for $L^{2}(-\pi, \pi)$. Then there exists a constant $\varepsilon>0$ with the following property: If $\left|\operatorname{Re} z_{n}-\lambda_{n}\right| \leqslant \varepsilon$ and $\sup \left|\operatorname{Im} z_{n}\right|<\infty$, then $\left\{e^{i z_{n} t}\right\}$ is also a Besselian basis for $L^{2}(-\pi, \pi)$.

2. On a theorem of Levinson. A well-known theorem of Levinson [8] states that the completeness $L^{p}$ of the set $\left\{e^{i \lambda_{n} t}\right\}$ is unaffected if some $\lambda_{n}$ is replaced by another number. An analogous result for Schauder bases follows immediately from the observation [1, p. 61] that the set $\left\{e^{i \lambda_{n} t}\right\}$ is complete on a given interval if and only if it is possible to approximate some function $e^{i \alpha t}$ other than those already present.

Proposition 1. If the set $\left\{e^{i \lambda_{n} t}\right\}$ is a Schauder basis for $L^{p}(-\pi, \pi), p \geqslant 1$, then it remains a Schauder basis if we replace any $\lambda_{n}$ by some other number.

Proof. Suppose that $e^{i \alpha t} \notin\left\{e^{i \lambda_{n} t}\right\}$. Fix $f$ in $L^{p}(-\pi, \pi)$ and write $f$ $=\sum c_{n} e^{i \lambda_{n} t}, e^{i \alpha t}=\sum d_{n} e^{i \lambda_{n} t}$. It is to be shown that $\left\{e^{i \lambda_{n} t}\right\}$ remains a Schauder basis when $e^{i \lambda_{k} t}$ is replaced by $e^{i \alpha t}$. First note that $d_{k}$ cannot vanish since $\left\{e^{i \lambda_{n} t}\right\}_{n \neq k}$ is not complete. Therefore, we can express first $e^{i \lambda_{k} t}$ and then $f$ in terms of $e^{i \alpha t}$ and $\left\{e^{i \lambda_{n} t}\right\}_{n \neq k}$. To establish uniqueness, suppose that

$$
0=\sum_{n \neq k} a_{n} e^{i \lambda_{n} t}+a_{k} e^{i \alpha t}
$$

Since $\left\{e^{i \lambda_{n} t}\right\}$ is a basis, it is enough to show that $a_{k}=0$. But this is clear, since otherwise we could express $e^{i \alpha t}$ in terms of $\left\{e^{i \lambda_{n} t}\right\}_{n \neq k}$ and hence conclude that $\left\{e^{i \lambda_{n} t}\right\}_{n \neq k}$ were complete.

To establish Theorem 1, we will need the following general stability criterion due to Boas (see [10]).

Proposition 2. Let $\left\{x_{n}\right\}$ and $\left\{y_{n}\right\}$ be sequences in a Banach space $X$. Suppose that there exists a constant $\theta, 0 \leqslant \theta<1$, such that the inequality

$$
\left\|\sum c_{n}\left(x_{n}-y_{n}\right)\right\| \leqslant \theta\left\|\sum c_{n} x_{n}\right\|
$$

holds for all finite sequences $\left\{c_{n}\right\}$. If $\left\{x_{n}\right\}$ is a (Besselian) basis for $X$, then $\left\{y_{n}\right\}$ is also a (Besselian) basis.

3. Proof of Theorem 1. Since $\left\{e^{i \lambda_{n} t}\right\}$ is a bounded basis for $L^{2}(-\pi, \pi)$, i.e. 


$$
0<\inf \left\|e^{i \lambda_{n} t}\right\| \leqslant \sup \left\|e^{i \lambda_{n} t}\right\|<\infty,
$$

we know that for some constant $\delta>0,\left\|e^{i \lambda_{n} t}-e^{i \lambda_{m} t}\right\| \geqslant \delta(n \neq m)[10, \mathrm{p}$. 22], and hence $\inf \left|\lambda_{n}-\lambda_{m}\right|>0(n \neq m)$. It follows [12] that

$$
\left\|\sum c_{n} e^{i \lambda_{n} t}\right\| \leqslant B\left(\sum\left|c_{n}\right|^{2}\right)^{1 / 2}
$$

for some constant $B$ and all finite sequences $\left\{c_{n}\right\}$. Now, it was shown in [6] that there exists a constant $p \geqslant 2$ with the following property: Each function $f$ belonging to $L^{2}(-\pi, \pi)$ has a unique expansion

$$
f=\sum c_{n} e^{i \lambda_{n} t} \text { with } A\left(\sum\left|c_{n}\right|^{p}\right)^{1 / p} \leqslant\|f\|,
$$

where $A>0$ is independent of $f$. It follows from (3) that for any finite sequence $\left\{c_{n}\right\}$,

$$
\begin{aligned}
\left\|\sum c_{n}\left(e^{i \lambda_{n} t}-e^{i \mu_{n} t}\right)\right\|=\left\|\sum c_{n} e^{i \lambda_{n} t}\left[e^{i\left(\mu_{n}-\lambda_{n}\right) t}-1\right]\right\| \\
=\left\|\left(\sum c_{n} e^{i \lambda_{n} t}\right) \sum_{k=1}^{\infty} \frac{i^{k} t^{k}\left(\mu_{n}-\lambda_{n}\right)^{k}}{k !}\right\| \\
=\left\|\sum_{k=1}^{\infty} \frac{i^{k} t^{k}}{k !} \sum c_{n}\left(\mu_{n}-\lambda_{n}\right)^{k} e^{i \lambda_{n} t}\right\| \\
\leqslant \sum_{k=1}^{\infty} \frac{\pi^{k}}{k !}\left\|\sum c_{n}\left(\mu_{n}-\lambda_{n}\right)^{k} e^{i \lambda_{n} t}\right\| \\
\leqslant B \sum_{k=1}^{\infty} \frac{\pi^{k}}{k !}\left\{\sum\left|c_{n}\left(\mu_{n}-\lambda_{n}\right)^{k}\right|^{2}\right\}^{1 / 2} .
\end{aligned}
$$

(The above calculations are similar to those used for an analogous purpose by Paley and Wiener [9].)

Now, if $p=2$, that is, if $\left\{e^{i \lambda_{n} t}\right\}$ is in fact Besselian, then the stronger Theorem 2 obtains. Let us assume therefore that $p>2$. Applying Hölder's inequality, we have

$$
\sum\left|c_{n}\left(\mu_{n}-\lambda_{n}\right)^{k}\right|^{2} \leqslant\left(\sum\left|c_{n}\right|^{p}\right)^{2 / p}\left(\sum\left|\lambda_{n}-\mu_{n}\right|^{2 k p /(p-2)}\right)^{(p-2) / p},
$$

and hence,

$$
\left\|\sum c_{n}\left(e^{i \lambda_{n} t}-e^{i \mu_{n} t}\right)\right\| \leqslant \theta\left\|\sum c_{n} e^{i \lambda_{n} t}\right\|
$$

where

$$
\begin{aligned}
\theta & =\frac{B}{A} \sum_{k=1}^{\infty} \frac{\pi^{k}}{k !}\left(\sum\left|\lambda_{n}-\mu_{n}\right|^{s}\right)^{k / s} \quad(s=2 p /(p-2)), \\
& =(B / A)\left[\exp \left\{\pi\left(\sum\left|\lambda_{n}-\mu_{n}\right|^{s}\right)^{1 / s}\right\}-1\right] .
\end{aligned}
$$


Since $s>2, N$ can be chosen large enough so that

$$
\left(\sum_{|n|>N}\left|\lambda_{n}-\mu_{n}\right|^{s}\right)^{1 / s}<\frac{1}{\pi} \log \left(1+\frac{A}{B}\right),
$$

and we conclude from Proposition 2 that the set $\left\{e^{i \lambda_{n} t}\right\}_{|n| \leqslant N} \cup\left\{e^{i \mu_{n} t}\right\}_{|n|>N}$ is a Schauder basis for $L^{2}(-\pi, \pi)$. Repeated application of Proposition 1 then shows that $\left\{e^{i \mu_{n} t}\right\}$ is itself a Schauder basis, and the proof is complete.

REMARK. For fixed $\left\{\lambda_{n}\right\}$, we have actually proved the stronger result that $\left\{e^{i \mu_{n} t}\right\}$ is a Schauder basis whenever $\sum\left|\lambda_{n}-\mu_{n}\right|^{s}<\infty$, where $s>2$ depends on the choice of $\left\{\lambda_{n}\right\}$.

4. Interpolation in a classical Hilbert space of entire functions. Let us denote by $H$ the Paley-Wiener space of entire functions of exponential type $\pi$ which are square-integrable over the real axis. A classical theorem of Paley and Wiener [9] shows that a function $f \in H$ if and only if $f=\hat{\varphi}$ for some function $\varphi$ in $L^{2}(-\pi, \pi)$. Here, as usual, $\hat{\varphi}$ denotes the complex Fourier transform of $\varphi$. A sequence $\left\{\lambda_{n}\right\}$ of distinct real or complex numbers is said to be an interpolating sequence for $H$ if $T H \supset l^{2}$, where $T$ is defined by $T f=\left\{f\left(\lambda_{n}\right)\right\}$. If the corresponding set of exponentials $\left\{e^{i \lambda_{n} t}\right\}$ is complete in $L^{2}(-\pi, \pi)$, then the interpolation is unique, and in this case we call $\left\{\lambda_{n}\right\}$ a complete interpolating sequence. The following proposition shows that the notions of complete interpolating sequences and Besselian bases are equivalent.

Proposition 3. Let $\left\{\lambda_{n}\right\}$ be a sequence of points lying in a strip parallel to the real axis. Then $\left\{\lambda_{n}\right\}$ is a complete interpolating sequence for $H$ if and only if $\left\{e^{i \lambda_{n} t}\right\}$ is a Besselian basis for $L^{2}(-\pi, \pi)$.

Proof. Suppose first that $\left\{e^{i \lambda_{n} t}\right\}$ is a Besselian basis for $L^{2}(-\pi, \pi)$. It follows from inequality (1) that the mapping from $l^{2}$ into $L^{2}(-\pi, \pi)$ given by $\left\{c_{n}\right\} \rightarrow \sum c_{n} e^{i \lambda_{n} t}$ is continuous, one-to-one, and onto. The open mapping theorem then guarantees the existence of a constant $A>0$ such that

$$
A\left(\sum\left|c_{n}\right|^{2}\right)^{1 / 2} \leqslant\left\|\sum c_{n} e^{i \lambda_{n} t}\right\|
$$

for all finite sequences $\left\{c_{n}\right\}$. But this is precisely the condition that ensures that $\left\{\lambda_{n}\right\}$ is an interpolating sequence for $H$ [2]. That the interpolation is unique follows immediately from the fact that $\left\{e^{i \lambda_{n} t}\right\}$ is a basis.

That any complete interpolating sequence $\left\{\lambda_{n}\right\}$, with the imaginary part of $\lambda_{n}$ uniformly bounded, determines a Besselian basis $\left\{e^{i \lambda_{n} t}\right\}$ was proved in [11].

5. Proof of Theorem 2. We conclude, just as in the proof of Theorem 1, that the inequality

$$
\left\|\sum c_{n}\left(e^{i \lambda_{n} t}-e^{i \mu_{n} t}\right)\right\| \leqslant B \sum_{k=1}^{\infty} \frac{\pi^{k}}{k !}\left[\sum\left|c_{n}\left(\mu_{n}-\lambda_{n}\right)^{k}\right|^{2}\right]^{1 / 2}
$$

obtains for some constant $B$ and all finite sequences $\left\{c_{n}\right\}$. Suppose first that $\left|\mu_{n}-\lambda_{n}\right| \leqslant \varepsilon$. Then (4) becomes 


$$
\left\|\sum c_{n}\left(e^{i \lambda_{n} t}-e^{i \mu_{n} t}\right)\right\| \leqslant B\left(e^{\pi \varepsilon}-1\right)\left(\sum\left|c_{n}\right|^{2}\right)^{1 / 2} .
$$

Now, since $\left\{e^{i \lambda_{n} t}\right\}$ is Besselian, a simple application of the closed graph theorem shows that if $f \in L^{2}(-\pi, \pi)$ and has the representation $f=\sum c_{n} e^{i \lambda_{n} t}$, then $\|f\| \geqslant A\left(\sum\left|c_{n}\right|^{2}\right)^{1 / 2}$ for some constant $A>0$ independent of $f[10, \mathrm{p}$. 338]. Thus, we have

$$
\left\|\sum c_{n}\left(e^{i \lambda_{n} t}-e^{i \mu_{n} t}\right)\right\| \leqslant(B / A)\left(e^{\pi \varepsilon}-1\right)\left\|\sum c_{n} e^{i \lambda_{n} t}\right\|
$$

for all finite sequences $\left\{c_{n}\right\}$. It follows immediately from Propositions 2 and 3 that $\left\{\mu_{n}\right\}$ is a complete interpolating sequence for $H$ whenever $\varepsilon$ is sufficiently small. Finally, it was shown in [11] that if the imaginary part of $z_{n}$ is uniformly bounded and if $\left\{\operatorname{Re} z_{n}\right\}$ is a complete interpolating sequence for $H$, then so too is $\left\{z_{n}\right\}$. A final application of Proposition 3 shows that $\left\{e^{i z_{n} t}\right\}$ is a Besselian basis for $L^{2}(-\pi, \pi)$, and the proof is complete.

REMARK. The proof of Theorem 2 has shown that, even if the $\lambda_{n}$ are not real, one can still choose $\varepsilon>0$ small enough so that $\left\{e^{i \mu_{n} t}\right\}$ is a Besselian basis for $L^{2}(-\pi, \pi)$ whenever $\left|\lambda_{n}-\mu_{n}\right| \leqslant \varepsilon$.

\section{REFERENCES}

1. W. Alexander and R. Redheffer, The excess of sets of complex exponentials, Duke Math. J. 34 (1967), 59-72. MR 34 \#6432.

2. N. K. Bari, Biorthogonal systems and bases in Hilbert space, Moskov. Gos. Univ. Učen. Zap. 148 Mat. 4 (1951), 69-107. (Russian) MR 14, 289.

3. R. Duffin and J. Eachus, Some notes on an expansion theorem of Paley and Wiener, Bull. Amer. Math. Soc. 48 (1942), 850-855. MR 4, 97.

4. R. Duffin and A. Schaeffer, A class of nonharmonic Fourier series, Trans. Amer. Math. Soc. 72 (1952), 341-366. MR 13, 839.

5. J. Elsner, Zulässige Abänderungen von Exponential-systemen im $L^{p}(-A, A)$, Math. Z. 120 (1971), 211-220.

6. V. Gurarii and N. Gurariī, Bases in uniformly convex and uniformly flattened Banach spaces, Izv. Akad. Nauk SSSR Ser. Mat. 35 (1971), 210-215 = Math. USSR Izv. 5 (1971), 220-225. MR 44 \#80.

7. M. Kadec, The exact value of the Paley-Wiener constant, Dokl. Akad. Nauk SSSR 155 (1964), 1253-1254 = Soviet Math. Dokl. 5 (1964), 559-561. MR 28 \#5289.

8. N. Levinson, Gap and density theorems, Amer. Math. Soc. Colloq. Publ., vol. 26, Amer. Math. Soc., Providence, R.I., 1940. MR 2, 180.

9. R. Paley and N. Wiener, Fourier transforms in the complex domain, Amer. Math. Soc. Colloq. Publ., vol. 9, Amer. Math. Soc., Providence, R. I., 1934.

10. I. Singer, Bases in Banach spaces. I, Die Grundlehren der math. Wissenschaften, Band 154, Springer-Verlag, New York and Berlin, 1970. MR 45 \#7451.

11. R. Young, Interpolation in a classical Hilbert space of entire functions, Trans. Amer. Math. Soc. 192 (1974), 97-114. MR 50 \#10290.

12. - An extension of the Hausdorff-Young theorem, Proc. Amer. Math. Soc. 45 (1974), $235-236$.

13. - A note on a trigonometric moment problem, Proc. Amer. Math. Soc. 49 (1975), $411-415$.

14. On perturbing bases of complex exponentials in $L^{2}(-\pi, \pi)$, Proc. Amer. Math. Soc. 53 (1975), 137-140. 